\title{
Quick introduction into AdS/CFT correspondence in physics of strongly correlated systems
}

\author{
M. B. Belonenko ${ }^{1,2}$, N. N. Konobeeva ${ }^{2}$, E. N. Galkina ${ }^{1,3}$ \\ ${ }^{1}$ Laboratory of Nanotechnology, Volgograd Institute of Business, 400048, Volgograd, Russia \\ ${ }^{2}$ Volgograd State University, 400062, Volgograd, Russia \\ ${ }^{3}$ Volgograd State Medical University, 400131, Volgograd, Russia \\ mbelonenko@yandex.ru, yana_nn@inbox.ru,galkina@mail.com
}

PACS 71.10-Ay, 71.27.+a

DOI 10.17586/2220-8054-2016-7-3-410-421

\begin{abstract}
The basic ideas of the AdS/CFT correspondence in physics of strongly correlated systems are briefly discussed. The application of the AdS/CFT correspondence for the Green's functions derivation are shown.
\end{abstract}

Keywords: AdS/CFT correspondence, Green's function.

Received: 22 January 2016

\section{Introduction}

The AdS/CFT correspondence appeared at the end of the 1990s as an approach established a connection between the string theory and conformal field theory models. In general, the existence of such relation could be expected if both model types have the same origin [1-8]. The evolution of hadron physics models has evolved in different ways over time. It was mandated by the fact that hadrons interact strongly, and in theory, there is no a small parameter on which it would be possible to make an expansion similar to the well-known quantum electrodynamics diagram approach. Initially, string theory was provided with phenomenological foundations in the form of Regge trajectories and conformal field theory got its essential evolution as a consequence of the phase transitions theory development [9]. When coming close to the phase-transition point, the physical value fluctuations have an increasing characteristic length. This fact requires scale invariance, and thus, conformal transformations and conformal theories immediately appear. From this, further development of such an approach has allowed the establishment experimentally testable theory corollaries as well. Note that these theories were divergent from one another increasingly not only in corollaries, but also in mathematical techniques, until a certain consistency between their parameters were established.

The basic concept of the AdS/CFT correspondence is in the formulae establishing a correspondence for average values $[2,6,8,10-14]$ :

$$
\left\langle\exp \left\{i \int_{\partial M} \phi_{0} \widehat{O}\right\}\right\rangle=\exp \left\{i S_{c l}(\phi)\right\} .
$$

Indeed, formula (1) consists of a variety of conceptions which must be deciphered. Thus:

1. Formula (1) maintains that the average values of operators $\widehat{O}$ on the boundary of some manifold $\partial M$, which are taken with classic field values $\phi$ on the boundary of a manifold $\phi_{0}$, will be coincident with the imaginary exponential of classic field action $\phi$ in a volume of manifold .

2. Operators $\widehat{O}$ cannot be chosen randomly and must be dual with field values $\phi$ (this choice will be shown in the part 2).

3. Manifold in a certain sense must be "good", have a boundary (or boundaries) and have a dimensionality one greater than the space for which we are going to construct the Green's function.

4. The formula (1) was given for Minkowsky space. It is necessary to make the Wick rotation of time axis for Euclidean space often used in Green's temperature functions calculation.

Note that after the average values are estimated, the problem for the Green's functions calculation becomes amply obvious. For example, the following expression can be used for the retarded Green's function [15]:

$$
G^{R}(\bar{k})=-i \int d^{4} \bar{x} \theta(t)\langle[\widehat{O}(\bar{x}), \widehat{O}(0)]\rangle,
$$

where $\theta(t)$ is Heaviside function as usual. 
Summarizing what was stated above, we can say that the AdS/CFT correspondence is a method for Green's function calculation apart from formulae $(1,2)$ for further use of these functions in applications. It is important to emphasize that concepts of AdS/CFT correspondence are not only limited to Green's function construction, but can also be applied for direct calculation of kinetic coefficient relations (for example, the well-known relation between viscosity and entropy density $\eta / s=1 / 4 \pi)[3,4,16-25]$.

In accordance with the above-stated, we build a plan of the present mini review. In the second chapter it will be briefly specified what kind of operators $\widehat{O}$ are dual with fields $\phi$ and how to choose a manifold. In the third part of this paper, a detailed case study for the scalar field will be analyzed. The basic formulae for Green's function calculation for fermions will be given in the fourth chapter and examples of the main Green's functions will be given. In the fifth chapter, a summary of the major scientific works expanding the primary models will be presented.

\section{Choice of operators and space}

Here and subsequently, a convention will be applied in which Greek indices correspond to the conformal field theory space on the boundary and Latin indices accord with field theory space of dimension one unit greater.

The choice of operators, $\widehat{O}$, as noted above, is not spontaneous and determined by the fact that operators must be dual. Since the primary role in this theory is played by fields $\phi$, which are defined by their transformation property under coordinate transformations; it is easier to bring the correspondence in a tabular form (Table 1).

TABLE 1. Correspondence of the classic field theory operators to the boundary operators

\begin{tabular}{|c|c|c|}
\hline $\begin{array}{c}\text { Field properties under } \\
\text { transformations } \\
\text { of coordinates }\end{array}$ & $\begin{array}{c}\text { Classic field theory } \\
\text { in a bulk }\end{array}$ & Operator on the boundary \\
\hline scalar & $\phi$ & boson operator $\widehat{O}_{B}$ \\
\hline vector & $A_{a}$ & current operator $\widehat{J}^{\mu}$ \\
\hline second-rank tensor & $g_{a b}$ & energy operator $\widehat{T}^{\mu \nu}$ \\
\hline spinor & $\psi$ & fermion operator $\widehat{O}_{F}$ \\
\hline
\end{tabular}

Note that well-known Green's fermion and boson functions can be constructed directly for fermion and boson operators, but for applications, there are important relations which result from Kubo formulae.

Thus, under calculation of shear viscosity $(\eta)$, it is easier to use expressions which follow from Kubo theory:

$$
\begin{gathered}
\eta=-\lim _{\varpi \rightarrow 0} \frac{\operatorname{Im} \Xi(\varpi)}{\varpi}, \\
\Xi(\varpi)=-i \frac{\hbar}{v} \int d^{4} \bar{r} e^{i \varpi t} \theta(t)\left\langle\left[\Pi_{x y}(\bar{r}, t), \Pi_{x y}(0,0)\right]\right\rangle,
\end{gathered}
$$

where $v$ is a normalization volume, and $\Pi_{x y}(\bar{r}, t)$ is a momentum flow density. It is convenient to carry out an analysis by reference to the second-rank tensor and general energy operator $\widehat{T}^{\mu \nu}$ in this case. Similarly, we can use vector $A_{a}$ and current operator $\widehat{J}^{\mu}$ for Hall conductivity calculation.

The choice of a space plays no less important role than the operator choice. A fundamental point lies with the fact that at absolute zero, theories will be correspond to the classic De Sitter space.

$$
s^{2}=\frac{L^{2}}{z^{2}}\left(d t^{2}+d \bar{x}^{2}+d z^{2}\right)
$$

where $z$ is an additional coordinate (which will be used for corresponding boundary conditions), $t$ is a time coordinate, $\bar{x}$ are space coordinates, $L$ is a parameter. Here the euclidian case is written down but proceeding to Minkowsky space can be performed by Wick rotation. In the case of theories with non-zero temperature it is necessary to consider more complicated metrics, for instance, of the following form which corresponds to a black hole type solution:

$$
\begin{gathered}
d s^{2}=\frac{R^{2}}{z^{2}}\left(f(z) d t^{2}+d \bar{x}^{2}+d z^{2} / f(z)\right), \\
f(z)=1-\frac{z^{4}}{z_{h}^{4}}
\end{gathered}
$$


where $z_{h}$ is a parameter which is directly associated with Hawking's temperature $T$ :

$$
z_{h}=1 /(\pi T) \text {. }
$$

Note that the metric (5) is applicable for models with a scalar field. For models with fermions, vector fields and others, it is essential to use relevant solutions of the Einstein's equations generalized to additional spatial dimension with energy-momentum tensor introduced in field models [26-39].

As an example, we provide a solution corresponding to a model with fermions which will be used in the chapter 4:

$$
\begin{gathered}
d s^{2}=\frac{z^{2}}{R^{2}}\left(-f(z) d t^{2}+d \bar{x}^{2}\right)+\frac{R^{2}}{z^{2} f(z)} d z^{2}, \\
f(z)=1+\frac{Q^{2}}{z^{4}}-\frac{1+Q^{2}}{z^{3}}, \\
T=\frac{3-Q^{2}}{4 \pi} .
\end{gathered}
$$

\section{Generation of the Green's functions for scalar field}

Let us consider in more detail the generation of the Green's functions for scalar field in pursuance of the paper [40]. We start with the case of absolute zero and De Sitter space metric defined by (4). As is well-known, the action for scalar field can be given by the following expression:

$$
S=-\frac{1}{2} \int d^{d+1} x \sqrt{g}\left[g^{M N} \partial_{M} \varphi \partial_{N} \varphi+m^{2} \phi^{2}\right] .
$$

Now, the corresponding motion equation will take the form:

$$
\frac{1}{\sqrt{g}} \partial_{M}\left(\sqrt{g} g^{M N} \partial_{N} \phi\right)-m^{2} \phi=0 .
$$

It is convenient to detach instantly the dependence on additional coordinate $z$ :

$$
z^{d+1} \partial_{z}\left(z^{1-d} \partial_{z} \phi\right)+z^{2} \delta^{\mu \nu} \partial_{\mu} \partial_{\nu} \phi-m^{2} L^{2} \phi=0
$$

Since the coefficients of equation do not explicitly depend on other coordinates, it is naturally to produce Fourier transform:

$$
\phi\left(z, x^{\mu}\right)=\int \frac{d^{d} k}{(2 \pi)^{d}} e^{i k x} f_{k}(z) .
$$

Next, we obtain the following expression for the Fourier coefficients:

$$
z^{d+1} \partial_{z}\left(z^{1-d} \partial_{z} f_{k}\right)-k^{2} z^{2} f_{k}-m^{2} L^{2} f_{k}=0
$$

Let us consider the given equation at a point $z=0$. Let us assume that $f_{k} \sim z^{\beta}$. Then, $\beta$ satisfies the following expression:

$$
\beta(\beta-d)-m^{2} L^{2}=0,
$$

where $d$ is the conformal theory space dimension. Solution of this equation is as follows:

$$
\beta=\frac{d}{2} \pm \sqrt{\frac{d^{2}}{4}+m^{2} L^{2}} .
$$

Thus, the solution near the border (it should be recalled that we construct the effective theory namely on the border) will be in the next form:

$$
\begin{aligned}
& f_{k}(z) \approx A(k) z^{d-\triangle}+B(k) z^{\triangle}, \\
& \triangle=\frac{d}{2}+\nu, \quad \nu=\sqrt{\frac{d^{2}}{4}+m^{2} L^{2}} .
\end{aligned}
$$

The relation (7) gives an opportunity to correlate the irregular on the border function $\phi$ with regular function $\varphi$ through the agency of the expression:

$$
\varphi(x)=\lim _{z \rightarrow 0} z^{\Delta-d} \phi(z, x) .
$$

After the brief introduction that gives an insight into both field behavior on the boundary and the prominent role of the parameter $\beta$ we can pass on to average calculation and the Green's function development. An average, 
as in any quantum theory, can be evaluated out from the following relation (it is important that we have to apply the fields $\phi$ which are defined on the boundary):

$$
\langle O(x)\rangle_{c}=\frac{\delta S_{g r a v}^{r e n}[\phi]}{\delta \varphi(x)}
$$

or to pass on to variation derivative in a bulk:

$$
\langle O(x)\rangle_{\varphi}=\lim _{z \rightarrow 0} z^{d-\triangle} \frac{\delta S_{g r a v}^{r e n}[\phi]}{\delta \phi(z, x)} .
$$

Hereafter, let us recall how to perform calculations with action in a bulk (index $M$ ) and on the boundary $(\partial)$ ):

$$
\begin{aligned}
S_{\text {grav }} & =\int_{\mathcal{M}} \int d z d^{d} x \mathcal{L}[\phi, \partial \phi], \\
\delta S_{\text {grav }} & =\int_{\mathcal{M}} \int d z d^{d} x\left[\frac{\partial \mathcal{L}}{\partial \phi} \delta \phi+\frac{\partial \mathcal{L}}{\partial\left(\partial_{\mu} \phi\right)} \delta\left(\partial_{\mu} \phi\right)\right], \\
\delta S_{\text {grav }} & =\int_{\mathcal{M}} \int d z d^{d} x\left[\left(\frac{\partial \mathcal{L}}{\partial \phi}-\partial_{\mu}\left(\frac{\partial \mathcal{L}}{\partial\left(\partial_{\mu} \phi\right)}\right)\right) \delta \phi+\partial_{\mu}\left(\frac{\partial \mathcal{L}}{\partial\left(\partial_{\mu} \phi\right)} \delta \phi\right)\right], \\
\delta S_{\text {grav }}^{\text {on }- \text { shell }} & =\int_{\varepsilon}^{\infty} \int d^{d} x \partial_{z}\left(\frac{\partial \mathcal{L}}{\partial\left(\partial_{z} \phi\right)} \delta \phi\right)=-\left.\int_{\partial M} d^{d} x \frac{\partial \mathcal{L}}{\partial\left(\partial_{z} \phi\right)} \delta \phi\right|_{z=\varepsilon} .
\end{aligned}
$$

It is convenient to introduce an analogue of a pulse having applied the additional coordinate:

$$
\Pi=-\frac{\partial \mathcal{L}}{\partial\left(\partial_{z} \phi\right)} \text {. }
$$

Now the calculation of variation derivatives can be simplified:

$$
\begin{aligned}
& \delta S_{\text {grav }}^{\text {on-shell }}=\int_{\partial M} d^{d} x \Pi(\epsilon, x) \delta \phi(\epsilon, x), \\
& \frac{\delta S_{\text {grav }}^{\text {on-shell }}}{\delta \phi(\epsilon, x)}=\Pi(\epsilon, x)=-\frac{\partial \mathcal{L}}{\partial\left(\partial_{z} \phi\right)} .
\end{aligned}
$$

In the general case, of course, there will be divergences in the action that is why it is necessary to introduce counterterms $S_{c t}$ :

$$
S^{\text {ren }}=S_{\text {grav }}^{o n-s h e l l}+S_{c t} .
$$

After that we should redefine the value $\Pi$ as well:

$$
\begin{aligned}
& \Pi^{r e n}(z, x)=\frac{\delta S^{r e n}}{\delta \phi(z, x)}, \\
& \Pi^{r e n}(z, x)=-\frac{\partial \mathcal{L}}{\partial\left(\partial_{z} \phi(\epsilon, x)\right)}+\frac{\delta S_{c t}}{\delta \phi(\epsilon, x)} .
\end{aligned}
$$

Finally, we can get the expression for average values calculation:

$$
\langle O(x)\rangle_{\varphi}=\lim _{z \rightarrow 0} z^{d-\triangle} \Pi^{r e n}(z, x) .
$$

Now, we pass on to direct calculation of the Green's functions. Recall that:

$$
\langle O(x)\rangle_{\varphi}=\int[D \psi] O(x) \exp \left(S_{E}[\psi]+\int d^{d} y \varphi(y) O(y)\right) .
$$

Hereinafter we write an evident expansion:

$$
\langle O(x)\rangle_{\varphi}=\langle O(x)\rangle_{\varphi=0}+\int d^{d} y\langle O(x) O(y)\rangle \varphi(y)+\ldots
$$

Determining the Green's functions as follows:

$$
G_{E}(x-y)=\langle O(x) O(y)\rangle,
$$


we can rewrite the expression (9) in the form:

$$
\begin{aligned}
\langle O(x)\rangle_{\varphi} & =\langle O(x)\rangle_{\varphi=0}+\int d^{d} y G_{E}(x-y) \varphi(y), \\
\langle O(x)\rangle_{\varphi} & =\int d^{d} y G_{E}(x-y) \varphi(y)
\end{aligned}
$$

or after performing a Fourier transform we get:

$$
\begin{aligned}
\langle O(k)\rangle_{\varphi} & =G_{E}(k) \varphi(k), \\
G_{E}(k) & =\frac{\langle O(k)\rangle_{\varphi}}{\varphi(k)} .
\end{aligned}
$$

Now using (8) we can obtain the final expression for the Green's function:

$$
G_{E}(k)=\lim _{z \rightarrow 0} z^{2(d-\triangle)} \frac{\Pi^{r e n}(z, x)}{\phi(z, k)} .
$$

We will meet a similar expression futher in the ratio for Green's fermionic function. Note that such structure remains for the Green's functions of fields with other spin value.

Next remember the equation for scalar field action:

$$
S=-\frac{\eta}{2} \int d z d^{d} x \sqrt{g}\left[g^{M N} \partial_{M} \phi \partial_{N} \phi+m^{2} \phi^{2}\right] .
$$

After discrimination of the total derivative the equation becomes as follows:

$$
S=-\frac{\eta}{2} \int d z d^{d} x \partial_{M}\left[\sqrt{g} \phi g^{M N} \partial_{N} \phi\right]+\frac{\eta}{2} \int d z d^{d} x \phi \sqrt{g}\left[\frac{1}{\sqrt{g}} \partial_{M}\left(\sqrt{g} g^{M N} \partial_{N} \phi\right)-m^{2} \phi\right] .
$$

Now the action on the boundary of the manifold takes the form:

$$
\begin{aligned}
S^{o n-s h e l l} & =-\frac{\eta}{2} \int d z d^{d} x \partial_{M}\left[\sqrt{g} \phi g^{M N} \partial_{N} \phi\right], \\
S^{o n-s h e l l} & =\frac{\eta}{2} \int d^{d}\left(\sqrt{g} \phi g^{z z} \partial_{z} \phi\right)_{z=\epsilon} \cdot
\end{aligned}
$$

Taking into account the expression for $\Pi$ :

$$
\Pi=-\frac{\partial \mathcal{L}}{\partial\left(\partial_{z} \phi\right)}=\eta \sqrt{g} g^{z z} \partial_{z} \phi
$$

it is easy to get that:

$$
S^{\text {on-shell }}=\frac{1}{2} \int_{z=\epsilon} d^{d} x \Pi(z, x) \phi(z, x)
$$

If we use the ratios for Fourier components:

we get that:

$$
\phi(z, x)=\int \frac{d^{d} k}{(2 \pi)^{d}} e^{i k x} f_{k}(z), \quad \Pi(z, x)=\int \frac{d^{d} k}{(2 \pi)^{d}} e^{i k x} \Pi_{k}(z),
$$

$$
S^{\text {on-shell }}=\frac{1}{2} \int \frac{d^{d} k}{(2 \pi)^{d}} \Pi_{-k}(z=\epsilon) f_{k}(z=\epsilon) .
$$

Refreshing the memory about $f$ on the boundary, it is easy to comprehend that on the boundary $\Pi$ behaves like:

$$
\Pi(z, x) \approx \eta L^{d-1}\left[(d-\triangle) A(x) z^{-\triangle}+\triangle B(x) z^{\triangle-d}\right], \quad(z \rightarrow 0) .
$$

The same expression in Fourier components will be as follows:

$$
\Pi_{-k}(z) \approx \eta L^{d-1}\left[(d-\triangle) A(-k) z^{-\triangle}+\triangle B(-k) z^{\triangle-d}\right], \quad(z \rightarrow 0) .
$$

This makes it possible to rewrite the expression for the action on the boundary:

$$
S^{\text {on-shell }}=\frac{\eta}{2} L^{d-1} \int \frac{d^{d} k}{(2 \pi)^{d}}\left[\epsilon^{-2 \nu}(d-\triangle) A(-k)+d A(-k) B(k)\right] .
$$


Unfortunately, this action diverges (see the power of $\varepsilon$ ). It is necessary to add a counterterm to the action for its regularization. Upon the demand for invariance and simplicity an appropriate counterterm will be as follows:

$$
\int_{\partial A d S} d^{d} x \sqrt{\gamma} \phi^{2}(\epsilon, x)
$$

where:

$$
d s_{z=\epsilon}^{2}=\gamma_{\mu \nu} d x^{\mu} d x^{\nu}=\frac{L^{2}}{\epsilon^{2}} \delta_{\mu \nu} d x^{\mu} d x^{\nu}
$$

It is easy to modify the counterterm and we can see that:

$$
\int_{\partial A d S} d^{d} x \sqrt{\gamma} \phi^{2}(\epsilon, x)=L^{d} \int \frac{d^{d} k}{(2 \pi)^{d}}\left[\epsilon^{-2 \nu} A(-k) A(k)+2 A(-k) B(k)\right] .
$$

For now, write down the counter term with correct coefficient and we obtain:

$$
\begin{aligned}
& s_{c t}=-\frac{\eta}{2} \frac{d-\triangle}{L} \int_{\partial A d S} d^{d} x \sqrt{\gamma} \phi^{2}, \\
& s_{c t}=-\frac{\eta}{2} \frac{d-\triangle}{L^{1-d}} \int \frac{d^{d} k}{(2 \pi)^{d}}\left[\epsilon^{-2 \nu} A(-k) A(k)+2 A(-k) B(k)\right] .
\end{aligned}
$$

From the last expression we finally obtain the formula for regularized action:

$$
S^{r e n}=\frac{\eta}{2} L^{d-1}(2 \triangle-d) \int \frac{d^{d} k}{(2 \pi)^{d}} A(-k) B(k) .
$$

Then let us represent the function $f$ as follows (for random $z$ );

$$
f_{k}(z)=A(k) \phi_{1}(z, k)+B(k) \phi_{2}(z, k) .
$$

It is naturally that for small $z$ we get:

$$
\phi_{1}(z, k) \approx z^{d-\triangle}, \quad \phi_{2}(z, k) \approx z^{\triangle} .
$$

Then, we introduce the following notation:

$$
\chi=\frac{B}{A}
$$

Now

$$
S^{r e n}=\frac{\eta}{2} L^{d-1}(2 \triangle-d) \int \frac{d^{d} k}{(2 \pi)^{d}} \chi(k) \varphi(k) \varphi(-k) .
$$

For now, it is easy to write an expression for variation derivative:

$$
\begin{aligned}
\langle O(x)\rangle_{\varphi} & =(2 \pi)^{d} \frac{\delta S^{r e n}}{\delta \varphi(-k)}=\eta L^{d-1}(2 \Delta-d) \chi(k) \varphi(k), \\
\langle O(k)\rangle_{\varphi} & =2 \nu \eta L^{d-1} B(k) .
\end{aligned}
$$

Wherefrom we immediately obtain that:

$$
G_{E}(k)=2 \nu \eta L^{d-1} \frac{B(k)}{A(k)} .
$$

This formula has an important significance and will be used hereafter and for fermions as well (but without formula derivation). Its meaning is in the fact that in the AdS/CFT correspondence for Green's function calculation it is just enough to determine coefficients at the equation's asymptotics with respect to additional coordinate for our field of interest:

$$
f_{k}(z)=z^{d / 2} g_{k}(z)
$$

Then:

$$
z^{2} \partial_{z}^{2} g_{k}+z \partial_{z} g_{k}-\left(\nu^{2}+k^{2} z^{2}\right) g_{k}=0
$$

This equation is precisely the equation for Bessel functions and its corresponding solution for $f$ will have a form $z^{d / 2} I_{\mp \nu}(k z)$.

Taking into account the fact that Bessel functions have the following asymptotic:

$$
I_{\mp \nu}(z) \approx \frac{1}{\Gamma(1 \mp \nu)}\left(\frac{z}{2}\right)^{\mp \nu}, \quad z \rightarrow 0,
$$


we can obtain that:

$$
\begin{gathered}
\phi_{1}(z, k)=\Gamma(1-\nu)\left(\frac{k}{2}\right)^{\nu} z^{d / 2} I_{-\nu}(k z), \quad \phi_{2}(z, k)=\Gamma(1+\nu)\left(\frac{k}{2}\right)^{-\nu} z^{d / 2} I_{\nu}(k z), \\
f_{k}(z)=z^{d / 2}\left[\Gamma(1-\nu)\left(\frac{k}{2}\right)^{\nu} A(k) I_{-\nu}(k z)+\Gamma(1+\nu)\left(\frac{k}{2}\right)^{-\nu} B(k) I_{\nu}(k z)\right] .
\end{gathered}
$$

Correspondingly:

$$
I_{\mp \nu}(z) \approx \frac{e^{z}}{\sqrt{2 \pi z}}, \quad(z \rightarrow \infty) .
$$

Now we have that:

$$
f_{k}(z) \approx \frac{z^{d / 2} e^{k z}}{\sqrt{2 \pi k z}}\left[\Gamma(1-\nu)\left(\frac{k}{2}\right)^{\nu} A(k)+\Gamma(1+\nu)\left(\frac{k}{2}\right)^{-\nu} B(k)\right] .
$$

From which,we get:

$$
\frac{B(k)}{A(k)}=-\frac{\Gamma(1-\nu)}{\Gamma(1+\nu)}\left(\frac{k}{2}\right)^{2 \nu}=\frac{\Gamma(-\nu)}{\Gamma(\nu)}\left(\frac{k}{2}\right)^{2 \nu} .
$$

Finally, the expression for the scalar field Green's function take the form:

$$
G_{E}(k)=2 \nu \eta L^{d-1} \frac{\Gamma(-\nu)}{\Gamma(\nu)}\left(\frac{k}{2}\right)^{2 \nu}
$$

or by performing an inverse Fourier transform, we derive:

$$
G_{E}(x)=\int \frac{d^{d} k}{(2 \pi)^{d}} e^{i k x} G_{E}(k) .
$$

With account of the following:

$$
\int \frac{d^{d} k}{(2 \pi)^{d}} e^{i k x} k^{n}=\frac{2^{n}}{\pi^{d / 2}} \frac{\Gamma\left(\frac{d+n}{2}\right)}{\Gamma\left(-\frac{n}{2}\right)} \frac{1}{|x|^{d+n}} .
$$

We derive a resultant expression for average with which we have defined the Green's function.

$$
\langle O(x) O(0)\rangle=\frac{2 \nu \eta L^{d-1}}{\pi^{d / 2}} \frac{\Gamma\left(\frac{d}{2}+\nu\right)}{\Gamma(-\nu)} \frac{1}{|x|^{2 \Delta}} .
$$

Note that the expression for the Green's function has the well known conformal field theory form with a power law of decrease. From the given methodical example, it is possible to retrace what is necessary to do in more complex cases.

\section{Green's functions for fermion field}

In this case, it is necessary to start with known models [41] that consist of not only fermion fields but also metric solutions in which a boundary naturally arises. An eligible candidate is a solution in $\mathrm{AdS}_{5}$ for the charged black hole in IIB supergravity type with three $U(1)$ charge, wherein $Q 1=Q 2$ and $Q 3=0$.

The Lagrangian density for this model is:

$$
\mathcal{L}=\frac{1}{2 k^{2}}\left\{R-\frac{1}{4} e^{4 \alpha} F_{\mu \nu} F^{\mu \nu}-12 \partial_{\mu} \alpha \partial^{\mu} \alpha+\frac{1}{L^{2}}\left(8 e^{2 \alpha}+4 e^{-4 \alpha}\right)\right\} .
$$


Here, $k$ is a coupling constant, $R$ is a crookedness, $F_{\mu \nu}$ is a density tensor of a vector field $A_{\mu}, \alpha$ is a scalar field. In this case, the solution for metrics, scalar and vector fields will have the following form:

$$
\begin{aligned}
& d s^{2}=e^{2 A}\left(-h d t^{2}+d x^{2}\right)+\frac{e^{2 B}}{h} d r^{2}, \\
& A=\ln \frac{r}{L}+\frac{1}{3} \ln \left(1+\frac{Q^{2}}{r^{2}}\right), \\
& B=-\ln \frac{r}{L}-\frac{2}{3} \ln \left(1+\frac{Q^{2}}{r^{2}}\right), \\
& h=\frac{\left(r^{2}+2 Q^{2}\right) r^{2}}{\left(r^{2}+Q^{2}\right)^{2}}, \quad \alpha=\frac{1}{6} \ln \left(1+\frac{Q^{2}}{r^{2}}\right), \\
& A_{\mu} d x^{\mu}=\Phi d t, \quad \Phi=\frac{\sqrt{2} Q r^{2}}{\left(r^{2}+Q^{2}\right) L},
\end{aligned}
$$

where $r$ is a radial coordinate, $Q$ is a black hole charge. Since the metrics is diagonal and depends only on $r$ Dirac equations can be simplified by substitution:

We write down Dirac equation for fermions:

$$
\begin{gathered}
\Psi \rightarrow\left(-g g^{r r}\right)^{1 / 4} \Psi \\
g=\operatorname{det}\left(g_{\alpha \beta}\right) .
\end{gathered}
$$

$$
\left[\gamma^{\mu}\left(\partial_{\mu}-i q A_{\mu}\right)-m\right] \psi=0
$$

here $q$ and $m$ are charge and mass as well, $\gamma$ are Dirac matrices. Then, after separation of variables, on which the equation coefficients don’t depend, we get:

It is easy to obtain that:

$$
\psi \rightarrow e^{-i w t+i k x} \psi
$$

$$
\left[-i \sqrt{-g^{t t}} \gamma^{t}\left(w+q A_{t}\right)+\sqrt{g^{r r}} \gamma^{r} \partial_{r}+i \sqrt{g^{x x}} \gamma^{x} k-m\right] \Psi=0
$$

As in the usual Dirac theory, these equations are split into pairs. Writing through the Pauli matrices $\sigma$ one pair of equations we have:

$$
\left[\sqrt{-g^{t t}} \sigma_{1}\left(w+q A_{t}\right)+\sqrt{g^{r r}} \sigma_{3} \partial_{r}+(-1)^{\alpha} i \sqrt{g^{x x}} \sigma_{2} k-m\right] \Psi_{\alpha}=0 .
$$

The asymptotic behavior of Dirac spinor in $\mathrm{AdS}_{5}$ has the following form:

$$
\psi_{\alpha} \stackrel{r \rightarrow \infty}{\longrightarrow} a_{\alpha} r^{m}\left(\begin{array}{l}
1 \\
0
\end{array}\right)+b_{\alpha} r^{-m}\left(\begin{array}{l}
0 \\
1
\end{array}\right) \text {. }
$$

The expected value of the boundary spinorial operator dual to the bulk spinor $\psi$ can be written as: $\left\langle O_{\psi}\right\rangle=$ $\left(0, b_{1}, 0, b_{2}\right)^{T}$. In fact, $O_{w}=0.5\left(1-\gamma^{\underline{r}}\right) O_{w}, \gamma^{\underline{r}}=\left(\begin{array}{cc}\sigma_{3} & 0 \\ 0 & \sigma_{3}\end{array}\right)$, which means that the boundary spinorial operator is left-handed, where $\sigma_{3}$ is the Pauli matrix. By imposing the in-falling boundary condition at the horizon, we can obtain the retarded Green's function as:

$$
G=\left(\begin{array}{cccc}
0 & & & \\
& G_{1} & & \\
& & 0 & \\
& & & G_{2}
\end{array}\right), \quad G_{\alpha}=\frac{b_{\alpha}}{a_{\alpha}} .
$$

Note that if we use the alternative quantization, the Green's function has the form: $G_{\alpha}=-a_{\alpha} / b_{\alpha}$, then the spinor operator at the border is right-handed. If $m=0, G_{1}$ and $G_{2}$ are related by $G_{2}=-1 / G_{1}$ [42]. Therefore, the alternative quantization for $G_{1}$ is the standard quantization for $G_{2}$, and vice versa. Taking $G_{1}$ and $G_{2}$ into account, the alternative quantization gives the same Fermi momenta as the standard quantization does at $m=0$.

The fact that the corresponding Green's functions in (14) are defined in the same manner as for the scalar field Green's functions in (11) has important significance. That is the general prescription of the Green's function construction for more complex theories as well. 
Let us consider equations for $\psi_{1}=\left(u_{1}, u_{2}\right)^{T}$ and introduce $u_{ \pm}=u_{1} \pm i u_{2}$, then we get:

$$
\begin{gathered}
\partial_{r} u_{+}+h^{*}(r) u_{+}=f^{*}(r) u_{-}, \\
\partial_{r} u_{-}+h(r) u_{-}=f(r) u_{+}, \\
h(r)=i \sqrt{\frac{-g^{t t}}{g^{r r}}}\left(w+q A_{t}\right), \\
f(r)=\frac{m}{\sqrt{g^{r r}}}-i k \sqrt{\frac{g^{x x}}{g^{t t}}} .
\end{gathered}
$$

From which, we finally obtain:

$$
\begin{gathered}
\partial_{r r} u_{+}+p^{*}(r) \partial_{r} u_{+}+q^{*}(r) u_{+}=0, \\
\partial_{r r} u_{-}+p(r) \partial_{r} u_{-}+q(r) u_{-}=0, \\
p(r)=-\partial_{r} f(r) / f(r), \\
q(r)=|h(r)|^{2}-|f(r)|^{2}+p(r) h(r)+h(r) .
\end{gathered}
$$

For massless particles $m=0$ and for the case when $w \propto 0(q>1 / 2)$, the solution can be written exactly:

$$
\begin{gathered}
u_{-}(r)=\left(\frac{r}{r+i \sqrt{2} Q}\right)^{\nu(k)}\left(\frac{r+i \sqrt{2} Q}{r-i \sqrt{2} Q}\right)^{q / 2}{ }_{2} F_{1}\left(\nu(k)-q+1 / 2, \nu(k) ; 2 \nu(k)+1 ; \frac{2 r}{r+i \sqrt{2} Q}\right), \\
\nu(k)=\frac{k}{\sqrt{2} Q} .
\end{gathered}
$$

Now, under $w \propto 0$, we finally have:

$$
G_{1}=\lim _{r \rightarrow \infty}\left(-i \frac{(-1)^{n+1} u_{-}^{*}-u_{-}}{(-1)^{n+1} u_{-}^{*}+u_{-}}\right), \quad n=q-\nu(k)-1 / 2 .
$$

Further calculations are performed, assuming that we have a precise analytical solution (15) for the case $w \propto 0$, we can develop perturbation theory and derive the Green's function in the ordinary form for calculations in the frame of AdS/CFT correspondence:

$$
\begin{gathered}
G(w, k)=\frac{Z}{-w+v_{F}\left(k-k_{F}\right)-c\left(k_{F}\right) w^{2 \nu(k)}}, \\
k_{F}=k_{F}(n)=\sqrt{2} Q(q-n-1 / 2), \quad n=0,1,2, \ldots,|q-1 / 2| .
\end{gathered}
$$

Note that the formula (16) predicts few Fermi surfaces for the case of charge $>1 / 2$.

The Green's functional form, similar to the cited (16), is a typical form for computation in the framework of AdS/CFT correspondence, and appears for other metrics as well $[43,44]$.

\section{Further development of the approach}

Further development of the AdS/CFT correspondence approaches proceeds in the same manner as the alreadyexisting solid state physics models' application, but also the way of further extension of both metric and space classes.

An approach for the Green's functions calculation in the frame of AdS/CFT correspondence was evolved in article [43], but for the case when metric was described by the metrics of the so-called Schrodinger black hole. Unfortunately, the authors could not retrieve a compact enough analytic expression for the fermions Green's function and it was thus investigated numerically.

Generalization of AdS/CFT correspondence onto the case of D-branes and the use of the given description for zero-point sound in strange metals investigation has been done in the paper [45]. This research obviously demonstrates that methods under development can also be extended to more complex mathematical objects.

Research issues important for various applications of Feynman propagators were discussed in [46]. Note that this paper is among one of the first on that subject.

In article [47], the application of AdS/CFT correspondence to study the transport in new-found Dirac semimetals was proposed $[48,49]$. The authors succeeded in obtaining agreement with experimental data and explain transport characteristics' behavior in the critical zone.

In [50], the authors completed the natural generalization of the AdS/CFT correspondence for the case of two interacting subsystems. In this instance, it was assumed that one subsystem consists of strongly interacting 
fermions, the Green's function of which can be found in the framework of the AdS/CFT correspondence. Another subsystem interacts with the first one quite weakly (i.e. it is possible to apply a standard diagram technique within the confines of the perturbation theory) and its Green's functions can be found by the perturbation theory.

Article [51] is dedicated to the application of the method under consideration to clarify the characteristics of so-called zero-point sound in metals. For example, in [52], the authors used the standard method to derive an expression for the zero-point sound parameters, having applied the Green's functions followed from the correspondence under discussion.

Investigation [53] is also of interest, as an approach to the Green's functions calculation in the presence of magnetic field was evolved in this work and prospects of this approach application in the Hall quantum effect physics were discussed.

In our opinion, issues surrounding the Green's function in an external magnetic field were more successfully defined in [54], in which the Green's function in an external magnetic field was obtained in the form similar to (16) with momentum quantization which is identical to Landau quantization and experimentally observed characteristics were estimated.

The important applications of cuprates physics were considered in publication [55], where an exploration of mode stability was performed and in general, the Green's function was shown to be stable and have the form similar to (16).

The possibility for AdS/CFT correspondence application in high-temperature superconducting cuprates physics was also considered in [56], where the appearance of mechanisms for Fermi arcs generation was investigated and the Green's functions were adduced with regard to strong quasiparticle decay.

The properties of universal thermal and electrical conductivity were investigated and research for ReissnerNordstrom black hole in arbitrary dimension was performed in the scientific work [57].

Article [58] is dedicated to the generalization of AdS/CFT correspondence approaches in the case of impurities presence in system. The ways of impurity introduction and their correct description within suggested approach were discussed.

Further description of impurities combined with semi-holographic approach was given in the paper [44]. Impurities were characterized as a subsystem weakly interacting with a system from which Green's functions can be determined using the AdS/CFT correspondence.

An important approach generalization for the case of an external periodic potential presence was done in article [59]. The results can be useful for analysis of the non-Fermi liquids' transport characteristics in external periodic fields created by superstructures, for instance.

Some research [60] was dedicated to the Fermi surface structure within the framework of the proposed approach. The polar motion of Green's functions was also explored.

Detailed mathematical analysis of evolving problems in the approach under consideration and investigation of important special cases were performed in [42]. Asymptotics of arising solutions and the case of final temperatures were also analyzed in detail in this paper.

The features of non-Fermi liquids' behavior and the unique characteristics of arising Green's functions were discussed in [61]. The behavior criteria of a non-Fermi electronic liquid were also mentioned there.

Non-Fermi liquids were also considered in [62]. This article is especially valuable because the authors adjusted the calculations (analytical and numerical) to obtain the excitations dispersion laws. In particular, it was shown that excitations with dispersion law $\omega \propto k^{2.09 \pm 0.01}$ exist in Non-Fermi liquids.

A significant model of Fermi liquid was analyzed in [63]. In this article, the Luttinger relation was shown to relate the area enclosed by the Fermi surfaces to the fermion density, which was derived from Gauss's Law for the bulk electric field. All low energy modes were demonstrated to be consistent with Landau's Fermi liquid theory.

AdS/CFT correspondence was generalized for the case of M-theory in [64]. The charge transport properties of $2+1$ dimensional conformal field theories at non-zero temperature were considered. For the theory with Abelian $U(1)$ charges, the action of particle-vortex duality on the hydrodynamic-to-collisionless crossover function was presented, leading to powerful functional constraints for self-dual theories.

The publication [65] was devoted to quantum dot investigation and consisted of a variety of model descriptions in which AdS/CFT correspondence ideas can be used. The Kondo lattice models were also considered; they can be described by mean-field theory obtained by mapping a quantum impurity coupled to a self-consistent environment. Such a theory yields a 'fractionalized Fermi liquid' phase of conduction electrons coupled to a critical spin liquid state, and is an attractive mean-field theory of strange metals.

The research [66] is also of interest, where a general hydrodynamic theory of transport was analyzed in the vicinity of superfluid-insulator transitions in two spatial dimensions described by "Lorentz"-invariant quantum 
critical points. The exact results for the null frequency transport coefficients for a supersymmetric conformal field theory, which is solvable by the AdS/CFT correspondence were also presented.

Propagation of the AdS/CFT correspondence ideas onto the case of the absolute zero phases of compressible quantum matter was investigated in [67]. Fermi surfaces with the singularities of Landau's Fermi liquid theory have several phases, while other Fermi surfaces have non-Fermi liquid singularities. Compressible phases found in models applicable to condensed matter systems were also argued to be present in models obtained by applying chemical potentials.

An important application to calculate the relation of entropy to viscosity is given in the [68]. The viscosity to entropy ratio has been shown to take on a very simple universal value in all gauge theories with the AdS/CFT correspondence. Investigators described the origin of this universal ratio, and focused on how it is modified by generic higher derivative corrections, corresponding to curvature corrections on the gravity side of the duality. In particular, certain curvature corrections are known to push the viscosity to entropy ratio below its universal value. This fact disproves a longstanding conjecture that such a universal value represents a strict lower bound for any fluid in nature.

Consideration of the ratio of entropy to viscosity is also given in [69]. The existence of a sound mode, which is described by hydrodynamics, even at energies much greater than the temperature, was shown. The authors explain how these and other properties of the field theory are consistent with those of a $(3+1)$-dimensional Landau Fermi liquid which is finely tuned to the Pomeranchuk critical point.

In article [70], the dynamics of few cycle optical pulses in non-Fermi liquid was considered. Energy spectrum of non-Fermi liquid was taken from the AdS/CFT correspondence. Conditions of quasi-particle excitation existence were defined. The non-Fermi liquid parameters' impact on the shape of the few cycle pulses was estimated.

Paper [71] can be mentioned as an example of another application of the approach under consideration. The indirect spin-spin interaction between impurities in a non-Fermi quantum liquid system is theoretically investigated in this paper. The poles of the Green's functions are shown to be responsible for the observed excitation spectra. Specifically, the anti-de Sitter/conformal field theory (AdS/CFT) correspondence is used to gain access to the analytical expressions of the Green's functions for our particular problem. For example, the current-voltage characteristic is determined by the imaginary part of the Green's function. Great progress in the Green's functions calculation for different systems was made by the AdS/CFT correspondence between the conformal field theory and the theory of superstrings [72].

Thus, the AdS/CFT correspondence is a powerful method to obtain Green's functions in systems of strongly interacting particles. The use of this method has made possible significant progress towards answering a number of modern physics most vital questions.

\section{Acknowledgements}

This work was supported by the Russian Foundation for Basic Research under project No. 16-32-00230.

\section{References}

[1] Casalderrey-Solana J., Liu H., et al. Gauge/String Duality, Hot QCD and Heavy Ion Collisions. 2011, ArXiv:1101.0618.

[2] Gubser S.S., Klebanov I.R., Polyakov A.M. Gauge theory correlators from noncritical string theory. Phys. Lett. B, 1998,428 , P. 105-114.

[3] Hartnoll S.A. Lectures on holographic methods for condensed matter physics. Class. Quant. Grav., $2009,26,224002$.

[4] McGreevy J. Holographic duality with a view toward many-body physics. Adv. High Energy Phys., $2010,723105$.

[5] D'Hoker E., Freedman D.Z. Supersymmetric gauge theories and the AdS/CFT correspondence. 2002, ArXiv: hep-th/0201253.

[6] Witten E. Anti-de Sitter space and holography. Adv. Theor. Math. Phys., 1998, 2, P. 253-291.

[7] Aharony O., Gubser S.S., et al. N field theories, string theory and gravity. Phys. Rept., 2000, 323, P. $183-386$.

[8] Maldacena J.M. The Large N limit of superconformal field theories and supergravity. Adv. Theor. Math. Phys., 1998, 2, P. $231-252$.

[9] Zee A. Quantum Field Theory in a Nutshell, Princeton University Press, Princeton, 2010. 608 pp.

[10] Balasubramanian V., Giddings S.B., Lawrence A. What do CFTs tell us about anti-de Sitter spacetimes. J. High Energy Phys., 1999, 03, 001.

[11] Balasubramanian V., Kraus P., Lawrence A., Trivedi S.P. Holographic probes of anti-de Sitter spacetimes. Phys. Rev. D, 1999, 59, 104021.

[12] Policastro G., Starinets A. On the absorption by near-extremal black branes. Nucl. Phys. B, 2001, 610, P. 117-143.

[13] Danielsson U.H., Keski-Vakkuri E., Kruczenski M. Vacua, propagators, and holographic probes in AdS/CFT. J. High Energy Phys., 1999, $01,002$.

[14] Balasubramanian V., Kraus P., Lawrence A. Bulk versus boundary dynamics in anti-de Sitter spacetime. Phys. Rev. D, 1999 , 59, 046003.

[15] Pak A.V., Belonenko M.B., Dyachkov P.N. Electrophysical properties of CNT. The augmented cylindrical wave method. Nanosystems: physics, chemistry, mathematics, 2013, 4(4), P. 564-569.

[16] Herzog C.P. Lectures on holographic superfluidity and superconductivity. J. Phys. A, 2009, 42, 343001.

[17] Cubrovic M., Zaanen J., Schalm K. String Theory, Quantum Phase Transitions and the Emergent Fermi-Liquid. Science, 2009, 325, P. 439-444.

[18] Lee S.S. A non-Fermi liquid from a charged black hole: a critical Fermi ball. Phys.Rev. D, 2009, 79, 086006.

[19] Senthil T., Vishwanath A., et al. Deconfined' quantum critical points. Science, 2004, 303, P. 1490-1494. 
[20] Senthil T., Balents L., et al. Quantumcriticality beyond the Landau-Ginzburg-Wilson paradigm. Phys. Rev. B, $2004,70,144407$.

[21] Sachdev S. Quantun magnetism and criticality. Nature Phys., 2008, 4, P.! 173-185.

[22] Jensen K., Kachru S., et al. Towards a holographic marginal Fermi liquid. Phys. Rev. D, 2011, 84, 126002.

[23] Hartnoll S.A. Horizons, holography and condensed matter. 2011, ArXiv:1106.4324.

[24] Liu H., McGreevy J., Vegh D. Non-fermi liquids from holography. Phys. Rev. D, 2011, 83, 065029.

[25] Horowitz G.T. Introduction to holographic superconductors. 2010, ArXiv:1002.1722.

[26] Iqbal N., Liu H. Real-time response in AdS/CFT with application to spinors. 2009, ArXiv: 0903.2596.

[27] Iqbal N., Liu H. Universality of the hydrodynamic limit in AdS/CFT and the membrane paradigm. Phys. Rev. D, $2009,79,025023$.

[28] Benfatto G., Gallavotti G. Perturbation Theory of the Fermi Surface in a quantum liquid. A general quasiparticle formalism and onedimensional systems. Journal of Statistical Physics, 1990, 59, P. 541-664.

[29] Shankar R. Statistical Mechanics and its Applications. Physica A, 1991, 177, P. 530-536.

[30] Polchinski J. Effective field theory and the Fermi surface. 1992, Arxiv: hep-th/9210046.

[31] Romans L.J. Supersymmetric, cold and lukewarm black holes in cosmological Einstein-Maxwell theory. Nucl. Phys. B, 1992, 383, P. 395-415.

[32] Bagger J., Lambert N. Gauge symmetry and supersymmetry of multiple M2-branes. JHEP, 2008, 02, P. 105.

[33] Gustavsson A. Algebraic Structures on Parallel M2-Branes. Nucl. Phys. B, 2009, 811, P. 66.

[34] Anderson P.W. Luttinger-liquid" behavior of the normal metallic state of the 2D Hubbard model. Phys. Rev. Lett., 1990, 64, 1839.

[35] Chamblin A., Emparan R., Johnson C.V., Myers R.C. Charged AdS black holes and catastrophic holography. Phys. Rev. D, 1999, 60, 064018.

[36] Varma C.M., Littlewood P.B., et al. Phenomenology of the normal state of Cu-O high-temperature superconductors. Phys. Rev. Lett., 1989, 63, 1996.

[37] Varma C.M., Nussinov Z., van Saarloos W. Singular or non-Fermi liquids. Physics Reports, 2002,361 (6), P. $267-417$.

[38] Shankar R. Renormalization-group approach to interacting fermions. Rev. Mod. Phys., 1994, 66, P. 129.

[39] Gauntlett J.P. Varela. O. Consistent Kaluza-Klein reductions for general supersymmetric AdS solutions. Phys. Rev. D, 2007 , 76, 126007.

[40] Ramallo A.V. Introduction to the AdS/CFT correspondence. 2013, ArXiv:1310.4319.

[41] Gubser S.S., Ren J. Analytic fermionic Green's functions from holography. Phys. Rev. D, 2012, 86, 046004.

[42] Faulkner T., Liu H., J. McGreevy, Vegh D. Emergent quantum criticality, Fermi surfaces, and AdS2. Phys. Rev. D, 2011, 83, 125002.

[43] Wang J. Schrodinger Fermi Liquids. Phys. Rev. D, 2014, 89 (4), 046008.

[44] Belonenko M.B., Konobeeva N.N., Galkina E.N. Dynamics of few cycle optical pulses in a non-Fermi liquid and AdS/CFT correspondence. Mod. Phys. Lett. B, 2015, 29 (19), 15500965.

[45] Dey P., Roy S. Zero sound in strange metals with hyperscaling violation from holography. Phys. Rev. D, $2013, \mathbf{8 8}, 046010$.

[46] Ryang S. The Hadamard function and the Feynman propagator in the AdS/CFT correspondence. Phys. Lett. B, 1999, 469, P. 87-95.

[47] Jacobs V.P.J., Vandoren S.J.G., Stoof H.T.C. Holographic interaction effects on transport in Dirac semimetals. Phys. Rev. B, 2014, 90, 045108.

[48] Wan X., Turner A.M., Vishwanath A., Savrasov S.Y. Opological semimetal and Fermi-arc surface states in the electronic structure of pyrochlore iridates. Phys. Rev. B, 2011, 83, 205101.

[49] Burkov A.A., Balents L. Weyl semimetal in a topological insulator multilayer. Phys. Rev. Lett., $2011,107,127205$.

[50] Faulkner T., Polchinski J. Semi-holographic Fermi liquid. JHEP, 2011, 06, P. 012.

[51] Hoyos-Badajoz C., O’Bannon A., Wu J.M.S. Zero Sound in Strange Metallic Holography. JHEP, $2010,1009,086$.

[52] Levitov L.S., Shitov A.V. Green's functions. Problems with solutions. Fizmatlit, Moscow, 2003, 392.

[53] Basu P., Yang He J., Mukherjee A., Shieh H.H. Holographic Non-Fermi Liquid in a Background Magnetic Field. Phys. Rev. D, 2010, 82, 044036.

[54] Gubankova E., Brill J., et al. Strongly Interacting Matter in Magnetic Fields. Springer, Berlin, $2013,553$.

[55] Edalati M., Leigh R.G., Lo K.W., Phillips P.W. Dynamical Gap and Cuprate-like Physics from Holography. Phys. Rev. D, 2011, 83, 046012.

[56] Vegh D. Fermi arcs from holography. 2010, Arxiv: 1007.0246.

[57] Jain S. Universal thermal and electrical conductivity from holography. JHEP, 2010, 1011, P. 092.

[58] Hashimoto K., Iizuka N. Impurities in Holography and Transport Coefficients. 2012, ArXiv:1207.4643.

[59] Chesler P., Lucas A., Sachdev S. Conformal field theories in a periodic potential: Results from holography and field theory. Phys. Rev. D, 2014, 89, 026005.

[60] Fan Z.-Y. Exact fermionic Green's functions from holograpny. 2014, ArXiv:1407.0104.

[61] Faulner T., Iqbal N., et al. Holographic non-Fermi-liquid fixed points. Philosophical transaction A, 2011,369, P. 1941.

[62] Liu H., McGreevy J., Vegh D. Non-Fermi liquids from holography. Phys. Rev. D, 2011, 83, 065029.

[63] Sachdev S. A model of a Fermi-liquid using gauge-gravity duality. Phys. Rev. D, 2011, 84, 066009.

[64] Herzog C.P., Kovtun P., Sachdev S., Son D.T. Quantum critical transport, duality, and M-theory. Phys. Rev. D, 2007, 75, 085020.

[65] Sachdev S. Strange metals and the AdS/CFT correspondence. J. Stat. Mech., 2010, 1011, 11022.

[66] Hartnoll S.A., Kovtun P.K., Mueller M., Sachdev S. Theory oft he Nernst effect near quantum phase transitions in condensed matter, and in dyonic black holes. Phys. Rev. B, 2007, 76, 144502.

[67] Huijse L., Sachdev S. Fermi surfaces and gauge-gravity duality. Phys. Rev. D, 2011, 84, 026001.

[68] Cremonini S. The shear viscosity to entropy ratio: A status report. Mod. Phys. Lett. B, 2011,25 (23), P. 1867-1888.

[69] Davison R.A., Goykhman M., Parnachev M. ADS/CFT and Landau-Fermi liquids. JHEP, 2014, 1407, P. 109.

[70] Konobeeva N.N., Belonenko M.B. Propagation of few cycle optical pulses in marginal Fermi liquid and ADS/CFT correspondence. Physica B: Condensed Matter, 2015, 478, P. 43-46.

[71] Zhukov A.V., Bouffanais R., Pak A.V., Belonenko M.B. Study of the indirect interaction in a non-Fermi liquid within the AdS/CFT correspondence framework. Mod. Phys. Lett. B, 2015, 29 (17), 1550081.

[72] Konobeeva N.N., Belonenko M.B. Tunneling current in carbon nanotubes with deep impurities. Nanosystems: physics, chemistry, mathematics, 2013, 4 (4), P. 555-558. 